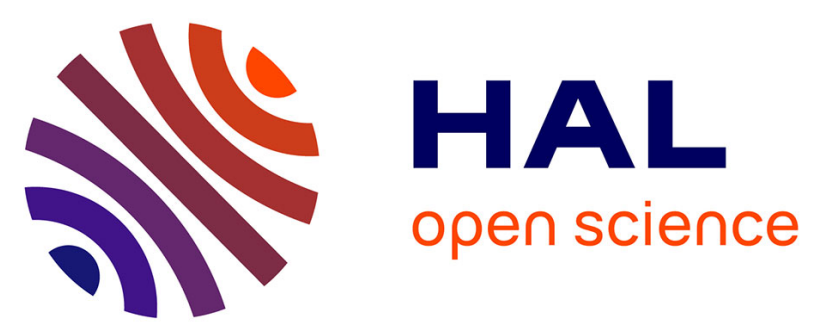

\title{
Saturation of Tungsten Surfaces with Hydrogen: A Density Functional Theory Study Complemented by Low Energy Ion Scattering and Direct Recoil Spectroscopy Data
}

\author{
Z.A. A Piazza, M. Ajmalghan, Y. Ferro, R.D. D Kolasinski
}

\section{To cite this version:}

Z.A. A Piazza, M. Ajmalghan, Y. Ferro, R.D. D Kolasinski. Saturation of Tungsten Surfaces with Hydrogen: A Density Functional Theory Study Complemented by Low Energy Ion Scattering and Direct Recoil Spectroscopy Data. Acta Materialia, 2018, 145, pp.388 - 398. 10.1016/j.actamat.2017.12.029 . hal-01793169

\section{HAL Id: hal-01793169 \\ https://hal.science/hal-01793169}

Submitted on 16 May 2018

HAL is a multi-disciplinary open access archive for the deposit and dissemination of scientific research documents, whether they are published or not. The documents may come from teaching and research institutions in France or abroad, or from public or private research centers.
L'archive ouverte pluridisciplinaire HAL, est destinée au dépôt et à la diffusion de documents scientifiques de niveau recherche, publiés ou non, émanant des établissements d'enseignement et de recherche français ou étrangers, des laboratoires publics ou privés. 


\title{
Saturation of Tungsten Surfaces with Hydrogen: A Density Functional Theory Study Complemented by Low Energy Ion Scattering and Direct Recoil Spectroscopy Data
}

\author{
Z. A. Piazza ${ }^{\mathrm{a}}$, M. Ajmalghan ${ }^{\mathrm{a}}$, Y. Ferro ${ }^{\mathrm{a},{ }^{*}}$ and R. D. Kolasinski ${ }^{\mathrm{b}}$ \\ ${ }^{a}$ Laboratoire PIIM, Aix-Marseille Université/CNRS, Avenue escadrille Normandie-Niemen, \\ 13397, Marseille, France \\ b Sandia National Laboratories, Energy Innovation Department, Livermore, California 94 \\ 551, USA
}

\begin{abstract}
Herein, we investigate the saturation limits of hydrogen on the (110) and (100) surfaces of tungsten via Density Functional Theory (DFT) and complement our findings with experimental measurements. We present a detailed study of the various stable configurations that hydrogen can adopt upon the surfaces at coverage ratios starting below 1.0, up to the point of their experimental coverage ratios, and beyond. Our findings allow us to estimate that the saturation limit on each surface exists with one monolayer of hydrogen atoms adsorbed. In the case of (110) this corresponds to a coverage ratio of one hydrogen atom per tungsten atom, while in the case of (100) a full monolayer is present at a coverage ratio of 2.0. Preliminary Low Energy Ion Scattering (LEIS) and Direct Recoil Spectroscopy (DRS) measurements complement these results and tend to confirm the findings obtained by DFT. In particular, the preferred adsorption sites on both surfaces at any coverage, the reconstruction of the (100) surface and the saturation limits agree well. We show that depending on the coverage, hydrogen surface binding energies can be of the same magnitude as binding energies to defects like vacancies. As a consequence, surface effects should be included in models aiming to simulate retention and desorption of hydrogen from the bulk.
\end{abstract}

Keywords: DFT, low energy ion spectroscopy, tungsten, hydrogen, surface

(*) Corresponding author: yves.ferro@univ-amu.fr 


\section{Introduction}

In this work, we provide a detailed DFT investigation of the adsorption of hydrogen on the (110) and (100) surfaces of tungsten. We started from the bare surface and adsorb hydrogen up to spontaneous desorption of a hydrogen molecule. At each step we examined various stable configurations of hydrogen and calculated their zero-point energy (ZPE) corrected adsorption energies. To complement the DFT study, our results are compared with low energy ion scattering (LEIS) and direct recoil spectroscopy (DRS) measurements.

The hydrogen-tungsten interaction is of high relevance in the field of nuclear fusion. Among the various materials to be used in a fusion reactor, plasma-facing components (PFCs) directly face the plasma and are consequently in strong interaction with hydrogen isotopes during runtime. Tungsten will be used as PFC in the next International Thermonuclear Experimental Reactor (ITER) currently built in Cadarache ${ }^{1}$. It also constitutes the JET-ILW divertor, ${ }^{2}$ where the interactions between the plasma and the wall are the strongest; tungsten is also the sole material used for the first wall material in the WEST tokamak.

The dissociation and recombination of $\mathrm{H}_{2}$ on various surfaces is a model system for catalysis, and indeed the dissociation of hydrogen on tungsten is directly related to the famous example of the formation of ammonia on top of tungsten surface used as catalyst. The elucidation of this mechanism was awarded the noble prize; it requires first the dissociation of the $\mathrm{N}_{2}$ and $\mathrm{H}_{2}$ molecules onto the $\mathrm{W}$ surface before $\mathrm{NH}_{3}$ is formed in several steps ${ }^{3,4}$.

From the experimental point of view, many studies were conducted on clean single crystalline samples. From LEED experiments ${ }^{5,6}$, the bare (100) surface of tungsten was shown to reconstruct below 370K. Hydrogen adsorption also induces a similar surface reconstruction of the tungsten atom as on the clean surface, which was also shown by LEED $^{7}$ experiments and ion scattering spectroscopy ${ }^{8}$. In particular, hydrogen was shown to form no island on top of the tungsten surface, but instead exhibited a homogenous distribution. Barnes and Willis ${ }^{9}$ 
showed that the surface reconstruction is strong at coverage below 0.5 , then tends to vanish while approaching the saturation limit, which they attribute to a coverage of 1.0 monolayer. Still based on LEED experiments, Barnes and Willis propose a structure for the reconstructed surface, which would be made of hydrogen located at Short-Bridge (SB) positions. The structure of the saturated surface with no reconstruction was recently resolved by means of Low Energy Ion Spectroscopy and Direct Recoil Spectroscopy ${ }^{10},{ }^{11}$ and is discussed later on in this paper.

Reconstruction is very small on the W(110) surface. Depending on the coverage, ordered phases were observed below $200 \mathrm{~K}$. They are the $\mathrm{p}(2 \times 1)$ at coverage 0.5 hydrogen atoms per surface tungsten atom, and the $\mathrm{p}(2 \times 2)$ at coverage $0.75^{12,13,14,15}$. The ordering of these two $\mathrm{p}(2 \times 1)$ and $\mathrm{p}(2 \times 2)$ phases however disappears above $200 \mathrm{~K}$ and $250 \mathrm{~K}$, respectively. Hydrogen was shown to prefer the Hollow site also called Three-Fold (TF) position ${ }^{15,16}$. However, due to the observation of phonon anomalies ${ }^{17}$ close to the saturation limit of hydrogen on the surface, the position of the hydrogen atom is still not completely clear while the structure of the ad-layer was determined to be a $\mathrm{p}(1 \times 1)$ phase $^{15}$. Based on EELS experiment, an Hinduced reconstruction of the tungsten surface atoms was also proposed ${ }^{18}$. This hypothesis was however debated after accurate measurements were performed by Balden and $a l^{15}$.

From the theoretical point of view, some previous calculations exist on both of the surfaces we investigate. On W(100), Heinola and Ahlgren ${ }^{19}$ calculated the adsorption of $\mathrm{H}$ on the clean unreconstructed surface via DFT. Their work shows that there are two stable positions for H, namely, the Long-Bridge (LB) and Short-Bridge (SB) positions; it was concluded that DFT predicts preferential adsorption of hydrogen at the SB position and this finding was corroborated by experimental results. Johnson and $\operatorname{Carter}^{20}$ found similar results by DFT as well as Moitra and Solanki ${ }^{21}$ on the clean W(100) surface. On the W(110) surface, Nojima 
and Yamashita ${ }^{22}$ established the stabilities of both the two $\mathrm{p}(2 \times 1)$ and $\mathrm{p}(2 \times 2)$ structures at coverage 0.5 and 0.75 , respectively. They showed that hydrogen prefers the TF position, in good agreement with other works based on $\mathrm{DFT}^{20,21}$. The penetration of hydrogen into the bulk via the $\mathrm{W}(100)^{19,20,21}$ and $\mathrm{W}(110)^{20,21,23,24}$ surfaces was also investigated by DFT, while the dissociation of molecular hydrogen was calculated via Molecular Dynamics (MD) calculations either based on $\mathrm{DFT}^{25}$ or on model potentials ${ }^{26,27}$. However, in all cases, these works were conducted on a clean $\mathrm{W}$ surface, hence modeling a very low coverage; this is in contrast with real experimental conditions. Only Nojima et $\mathrm{al}^{22}$ addressed more experimentally relevant coverage ratios of 0.5 and 0.75 on the W(110) surface, and Kolasinski et $\mathrm{al}^{10}$ performed calculations modeling a coverage of 1.0 monolayer on the $\mathrm{W}(100)$ surface.

To our knowledge, currently there are no systematic investigations of $\mathrm{H}$ adsorption on the $\mathrm{W}(110)$ and $\mathrm{W}(100)$ surfaces from the bare surfaces up to saturation point where $\mathrm{H}_{2}$ spontaneously desorbs. This topic is of high relevance to model the retention and release of hydrogen from tungsten. To this end, both thermodynamic ${ }^{28,29,30,31}$ and kinetic ${ }^{32,33,34,35,36,37}$ models built via DFT data exist, however, thus far surface effects have not yet been included with explicit physical ground. Both models aim to simulate and interpret experimental results, mostly from $\operatorname{TDS}^{3839,40,41,42,43,44}$. The contributions of this work are a first step toward understanding the relevant surface effects to be implemented in the larger scale models.

In light of this, our aim in the present work is to provide a detailed examination of hydrogen adsorption on the $\mathrm{W}(110)$ and $\mathrm{W}(100)$ surfaces starting from low coverage up to the point of spontaneous desorption of a hydrogen molecule. To complement this theoretical work, we compare our results with low energy ion beam experiments. At each coverage ratio, we determine the binding energy of hydrogen on the surface in order to assess the capability of 
the surface to act as a sink for hydrogen in tungsten. Our study is organized as follows: In section 2 we provide details of the DFT methodology we employed to construct periodic models of the surfaces and the equations we use to calculate hydrogen adsorption energies. In section 3 we investigate the adsorption of hydrogen on the $\mathrm{W}(110)$ and $\mathrm{W}(100)$ surfaces. Adsorption configurations of all coverage ratios permitted by the size of the unit-cells were calculated, and their relative stabilities at each coverage ratio are compared. In section 4, based on the most stable configurations we found, the saturation limit is defined for both surfaces and compared with experimental results. The impact of the surface on hydrogen retention in tungsten is discussed. In section 5, we present low energy ion scattering and direct recoil spectrometry measurements that enable direct determination of the $\mathrm{H}$ binding configuration on the surface. Finally, the primary conclusions of this work are given in section 6 .

\section{Computational Methods}

\section{1 - Electronic structure calculations}

In correspondence with a previous study on the interactions of hydrogen with bulk tungsten, herein we utilize a similar periodic plane-wave DFT model. We employ the $\mathrm{PBE}^{45}$ exchangecorrelation functional to calculate electronic energies. To describe the ionic cores we use the Vanderbilt ultra-soft scalar-relativistic ultra-soft pseudo-potentials ${ }^{46}$ (USPP); the valence and semi-core electrons were included for tungsten, specifically the $5 s^{2} 5 p^{6} 6 s^{2} 5 d^{4}$ electrons. Energy cutoffs of 40Ry and 320Ry were used for the wave-function and electron density, respectively. Converged k-point sampling schemes were chosen as described in Section 2.2 for tungsten (110) and (100) surfaces. Geometry optimizations were carried out until the forces on all atoms were less than a threshold of $0.0014 \mathrm{eV} / \AA ̊$. Vibrational frequencies of the hydrogen atoms were calculated via Density Functional Perturbation Theory (DFPT) ${ }^{47}$ while 
keeping all tungsten atoms frozen. Due to the mass difference of hydrogen and tungsten, this assumption is quite robust; It was shown in our previous work ${ }^{28}$ that at the current level of theory the difference in allowing the tungsten atoms to freely move in such phonon calculations results in deviations of less then $1 \mathrm{~cm}^{-1}$ for the vibrational energies of hydrogen atoms. More detail and justification for the applicability of this method to tungsten and hydrogen can be found in the previous study ${ }^{28}$. All of the DFT and DFPT calculations were carried out with the Quantum Espresso package ${ }^{48}$.

\subsection{Model of the surfaces}

Before investigating the adsorption of hydrogen onto tungsten, appropriate models of the bare tungsten (100) and (110) surfaces were developed. To do so, we created slab models and investigated the effect of the number of tungsten layers on the convergence of their calculated surface formation energies. In our previous work, it was shown that the primitive unit-vector for bulk tungsten using the current level of theory is $\mathbf{a}=3.187 \AA$ at zero temperature and pressure; the experimental value is $\mathbf{a}_{\exp }=3.165 \AA$. An $18 \times 18 \times 18 \mathrm{k}$-point sampling of the Brillouin Zone was shown large enough to converge the energy of the bulk unit-cell using the parameters described in Section 2.1. Accordingly, we used this information as a basis for our slab models.

We constructed tungsten $\mathrm{W}(110)$ surface $1 \times 1 \times \mathrm{Z}(\mathrm{Z}=2-8)$ slab models based on a primitive unit vector of $\mathbf{a}=3.187 \AA$ and included a $20.0 \AA$ vacuum in the $\mathrm{z}$-direction. Accordingly, our k-points sampling for the $\mathrm{W}(110)$ surfaces is $18 \times 13 \times 1$ since $\mathbf{b}=\mathbf{a} \sqrt{ } 2$, with $\mathbf{a}$ and $\mathbf{b}$ pointing toward the [001] and [110] directions, respectively. Since it is known that the bare tungsten W(100) surface is significantly reconstructed at low temperatures, we modeled both the reconstructed and non-reconstructed (100) surfaces with dimensions $2 \times 2 \times \mathrm{Z}$ with $\mathbf{a}$ and b pointing toward the [100] and [010] directions. Again we allowed the number of layers to vary and used a k-point sampling of $9 \times 9 \times 1$. Top-view illustrations of the basic tungsten 
(110) and (100) surface units are provided in Figure 1.

Each slab model was geometrically optimized and the surface formation energy was calculated as:

$$
S E=\frac{1}{2}\left(\frac{E_{\text {slab }}^{\text {DFT }}-N E_{\text {bulk }}^{D F T}}{A_{\text {surface }}}\right)
$$

where $E_{\text {slab }}^{D F T}$ is the total energy of the slab model, $N$ is the number of tungsten atoms in the slab model, and $E_{\text {bulk }}^{D F T}$ is the bulk energy per $\mathrm{W}$ atom. The equation is divided by two to account for the fact that the slab model has both a top and a bottom surface. For each slab model, calculated surface energies converged to an accuracy within $10 \mathrm{meV} / \AA^{2}$ within five layers (see Figure 2).

Accordingly, the models used in this work are composed of six layers of Tungsten with a vacuum of $20 \AA$. For all further calculations on each slab model we fixed the bottom two layers of tungsten atoms in the bulk geometry to directly simulate the bulk at this depth. The k-point sampling schemes were tested for each 6-layer slab model and found to be well converged upon comparison with more dense sampling schemes.

\subsection{Calculation of theoretical adsorption energies}

To describe the relative stability of various configurations of hydrogen on tungsten surfaces we calculated their adsorption energies as:

$$
E_{a d, H_{n}}=E_{W_{s l a b}, H_{n}}^{D F T}-E_{W_{s l a b}}^{D F T}-\frac{n}{2} E_{H_{2}}^{D F T}
$$

where $n$ is the number of hydrogen atoms, $E_{W_{s l a b}, H_{n}}^{D F T}$ is the energy value of a given configuration of $n$ hydrogen atoms on a tungsten surface ( $\left.\mathrm{W}_{\text {slab}}\right), E_{W_{\text {slab }}}^{D F T}$ is the energy of the corresponding bare surface, and $E_{\mathrm{H}_{2}}^{D F T}$ is the calculated value of molecular $\mathrm{H}_{2}$. A negative $\mathrm{E}_{\mathrm{ad}}$ reveals that a given configuration is theoretically stable at $0 \mathrm{~K}$. We also provide zero-point vibrational energy (ZPE) corrected values for all adsorption energies, labeled as $E_{a d, Z P E, H_{n}}$. 
Relative energy differences between various configurations at a given hydrogen coverage ratio (herein labeled by $\theta$ ) tell us which configurations are most stable for the given coverage. As the coverage ratio is increased, the change in the adsorption energy of the most stable configurations is calculated as:

$$
E_{a d}^{n+1}=E_{a d, Z P E, H_{n+1}}-E_{a d, Z P E, H_{n}}-\frac{1}{2} E_{H_{2}, Z P E}^{D F T}
$$

\section{Results}

\section{1 - Hydrogen adsorption on the W(110) surface}

Herein we use the symbol $\Theta$ to represent the ratio of hydrogen atom per surface tungsten atom $\left(\Theta=H / W_{\text {surface }}\right)$. This is done in order to alleviate the ambiguity of the symbol $\theta$ which is commonly defined in the surface science literature as $\theta=1.0$ when 1 layer of adsorbate surface sites are occupied, but does not in of itself explicitly state the stoichiometric ratio of adsorbate atoms to surface atoms.

A hydrogen to tungsten coverage of $\Theta=0.5$ on the $\mathrm{W}(110)$ surface represents a single hydrogen atom interacting with a single unit of the $1 \times 1 \times 6 \mathrm{~W}(110)$ surface model. This coverage has been studied previously via DFT ${ }^{22,49}$ where a three-fold (TF) site for hydrogen was found to be most stable. We found four elementary stationary sites of hydrogen on the tungsten W(110) surface, namely the hollow or three-fold (TF), short-bridge (SB), longbridge (LB), and top (T) positions; this is consistent with the work of Nojima et $\mathrm{al}^{22}$. Vibrational analysis reveals that all four sites are minima; this is in contrast to the previous DFT work where the LB and T sites were calculated as saddle points on the potential energy surface $^{22}$, possibly because the previous calculations were not fully converged to their minima on the potential energy surface. Table 1 provides top-view representations of each configuration along with their adsorption energies and some characteristic geometric data. 
The most stable position for a single hydrogen to occupy on the $\mathrm{W}(110)$ surface is the TF position with a calculated adsorption energy per hydrogen is $E_{a d, Z P E, H_{n}}=-0.70 \mathrm{eV}$. Upon adsorption of TF hydrogen atoms at coverage $\Theta=0.5$, the surface tungsten atoms are laterally shifted by $\approx 0.05 \AA$ in the $[1 \overline{1} 0]$ direction. This is a result of the interaction of tungsten and hydrogen and not a considerable surface reconstruction. Similarly, the second most stable configuration (SB) with an $E_{a d, Z P E, H_{n}}=-0.58 \mathrm{eV}$ induces a slight shift in the [11̄0] direction, while the less stable LB and $\mathrm{T}$ configurations do not cause a shift in the surface atoms.

At $\Theta=1.0$ we considered a large number of configurations combining the four elementary hydrogen positions and relaxing the geometry of each configuration. The occupation of $\mathrm{TF}$ sites is again favorable, followed by SB sites. In total, we found ten unique configurations of hydrogen adsorbed on the W(110) surface which have a negative $E_{a d, H_{n}}$. For the lowest energy configurations we also performed vibrational analysis to obtain the $E_{a d, Z P E, H_{n}}$ values (see Table 1). Top-view representations and $E_{a d, H_{n}}$ values of all ten configurations are given in the supplementary information. In the case of the lowest energy TF occupation at $\Theta=1.0$ with $E_{a d, Z P E, H_{n}}=-1.29 \mathrm{eV}$ there is one hydrogen atom in a TF position for each tungsten atom where the hydrogen atoms are maximally distant from one another. The $E_{a d}^{n+1}$ is -0.59 $\mathrm{eV}$, thus the adsorption of another $\mathrm{H}$ atom onto the $1 \times 1$ surface unit-cell relative to $\Theta=0.5$ is energetically favored. The hydrogen layer adopts exactly the same formation as the first $\mathrm{W}(110)$ sub-surface layer, but is offset laterally by $\approx 0.7 \AA$ in the $[1 \overline{1} 0]$ direction. The second most stable configuration at $\Theta=1.0$ is characterized by SB occupations along the diagonal of the unit-cell with an $E_{a d, Z P E, H_{n}}=-1.11 \mathrm{eV}$. This corresponds to maximally distant hydrogen atoms as compared to the other possible SB configurations at this coverage. 
To investigate configurations beyond $\Theta=1.0$ we followed a similar procedure, at each step,

of placing a new hydrogen atom at symmetrically unique TF, $\mathrm{SB}, \mathrm{LB}$, an $\mathrm{T}$ positions in the unit cell and performing geometry relaxations. For $\Theta=1.5$ we found nine unique configurations with a negative $E_{a d, H_{n}}$, for $\Theta=2.0$ we found 11 , and for $\Theta=2.5$ we found just one. The most stable configurations are presented in Table 2 along with their $E_{a d, Z P E, H_{n}}$ values, while the remaining configurations can be found in the supplementary information without ZPE corrected values. The most stable configuration at $\Theta=1.5$, with an $E_{a d, Z P E, H_{n}}=$ $-1.10 \mathrm{eV}$, can be described as adding hydrogen to one $\mathrm{T}$ position of the lowest-energy $\Theta=1.0$ coverage (all TF positions occupied). The $\mathrm{T}$ position hydrogen lies $\approx 1.7 \AA ̊$ above the tungsten surface and $\approx 0.7 \AA ̊$ above the mono-layer of TF position hydrogens. The overall configuration is stable at 0 Kelvin, but represents a decrease in stability as compared to the most favored configuration at $\Theta=1.0$ in accord with an $E_{a d}^{n+1}$ of $0.18 \mathrm{eV}$.

At $\Theta=2.0$, again, the most stable configuration formed is the result of adding a $\mathrm{T}$ position hydrogen, with an $E_{a d, Z P E, H_{n}}=-0.84 \mathrm{eV}$ and an $E_{a d}^{n+1}$ of $0.26 \mathrm{eV}$. The adsorption of another hydrogen atom to the surface represents a loss in stability relative to lower coverage. Geometrically, the configuration exhibits two layers of hydrogen with the same symmetry and spacing as the $\mathrm{W}(110)$ surface below; the first layer consists of hydrogen atoms in $\mathrm{TF}$ positions and lies $1.0 \AA$ above the surface, the second layer consists of $\mathrm{T}$ position hydrogens $1.7 \AA$ above the surface. The hydrogen atoms of the two respective layers are $1.8 \AA$ apart, which probably minimizes the interaction between the hydrogen atoms. The second most stable position with $\Theta=2.0$, has an $E_{a d, Z P E, H_{n}}=-1.03 \mathrm{eV}$ and is easily described as full, symmetric coverage of the $\mathrm{W}(110)$ surface by hydrogens at SB positions. Thus, we can see a bi-layered hydrogen system is favorable to a mono-layered system of coverage $\Theta=2.0$. 
The only stable configuration found at coverage of $\Theta=2.5$ strays from the growth pattern of adding a hydrogen to an open position on the most stable configuration of lesser coverage. The configuration is formed by adding two $\mathrm{T}$ hydrogens to the second most stable configuration at $\Theta=1.5$ (see Table 2). The two neighboring hydrogen atoms reside $0.93 \AA$ apart. The configuration has an $E_{a d, Z P E, H_{n}}=-0.65 \mathrm{eV}$. An $E_{a d}^{n+1}$ of $0.19 \mathrm{eV}$ show us that the trend which began at $\Theta=1.5$ of energetically disfavored adsorption of molecular hydrogen relative to a lower coverage ratio continues at $\Theta=2.5$.

At $\Theta=3.0$ we were not able to find any stable configurations. Many attempts to find such a configuration resulted in the combination of two hydrogen atoms forming $\mathrm{H}_{2}$ and desorbing from the surface with no activation barrier.

\section{2 - Hydrogen adsorption on the W(100) surface}

At low temperature, a surface reconstruction occurs for the tungsten W(100) surface with a $(\sqrt{2} \times \sqrt{2}) \mathrm{R} 45$ unit-cell and $p 2 m g$ symmetry, but it is known that the reconstruction disappears above $370 \mathrm{~K}^{5,6}$. This reconstruction of the $2 \times 2 \times 6 \mathrm{~W}(100)$ cell induces a stabilization in energy we calculated to $0.23 \mathrm{eV}$. We began by systematically adding hydrogen to the reconstructed $\mathrm{W}(100)$ surface to obtain the theoretically most stable configurations and to examine if the adsorption of hydrogen removes the surface reconstruction as the coverage ratio increases. In the following, adsorption energies of hydrogen atoms will be given with respect to the reconstructed surface.

Adding a single hydrogen to $2 \times 2 \times 6 \mathrm{~W}(100)$ surfaces represents a coverage of $\Theta=0.25$. For the reconstructed surface the only stable position available for hydrogen to adsorb above the surface are the short-bridge (SB) sites along the [110] direction and results in an $E_{a d, Z P E, H_{n}}=-0.82 \mathrm{eV}(-1.04 \mathrm{eV}$ with respect to the non reconstructed surface). This 
reconstruction is of the same type as the bare surface, but leads to a stabilization in energy that is four times the value. As a consequence, hydrogen strengthens the surface reconstruction that should persist at higher temperature. On the contrary to what was already published $^{19}$, we found no stable long-bridge (LB) position on top of the surface.

In order to reach $\Theta=0.5$ by adding another $\mathrm{H}$ atom to the surface, two SB possibilities exist. The first one is to have both the hydrogen atoms close to each other or to have them homogenously distributed along the [110] direction (Table 3). The former was shown to be the more stable by $0.16 \mathrm{eV}$.

Following this, hydrogen atoms were added up to a coverage of $\Theta=2.0$ with an increment of $\Delta \Theta=0.25$, at each step the only stable positions above the surface are SB positions. Once $\Theta=2.0$ is reached, all four available SB positions surrounding each tungsten atom are occupied, and the symmetry of the system effectively removes the surface reconstruction. Top-view configurational representations at $\Theta=0.5,1.0,1.5$ and 2.0 along with adsorption energies and characteristic geometric data are provided in Table 3.

The devolution of the surface reconstruction is quite clear visually; at $\Theta=1.0$, the reconstruction is obviously still present but noticeably lessened, by $\theta=1.5$ it is nearly lost, and by $\Theta=2.0$ the system is perfectly symmetric with no sign of the surface reconstruction remaining.

Energetically, The basic trend up to $\Theta=2.0$ is as follows: first SB hydrogen adsorbs to the surface with an $E_{a d, Z P E, H_{n}}=-0.82 \mathrm{eV}$ at $\Theta=0.25$. Then at each step after the first SB adsorption, the system is stabilized by $E_{a d}^{n+1} \approx-0.7 \mathrm{eV}$ (Table 3 ) quite linearly until there is 
full occupation at $\Theta=2.0$ of equivalent $\mathrm{SB}$ hydrogens roughly $1.0 \AA$ above the surface, constituting a hydrogen mono-layer. Once all SB positions have been occupied at $\Theta=2.0$ the $E_{a d, Z P E, H_{n}}=-5.50 \mathrm{eV}$ goes to a minimum in energy. The trend of $E_{a d}^{n+1}$ along with the minimum in $E_{a d, Z P E, H_{n}}$ suggests that adsorption of each subsequent molecular hydrogen is favored up to $\Theta=2.0$.

After coverage $\Theta=2.0$, there exist two types of stable positions where additional hydrogen atoms can adsorb, either directly on top (T) of a tungsten roughly $1.7 \AA$ above the surface or at positions more or less at the plane of the tungsten surface, laterally offset from the first subsurface layer which we refer to as Three Fold Position (TF), in each case there are four available positions on the $2 \times 2$ unit-cell. At a coverage of $\Theta=2.25$, the more stable configuration exists when an TF position is occupied, resulting in an $E_{a d, Z P E, H_{n}}=-5.49 \mathrm{eV}$, while the top position leads to $E_{a d, Z P E, H_{n}}=-5.29 \mathrm{eV}$. Thus, the SB occupation is $0.20 \mathrm{eV}$ more stable. Since the TF position is favored, we saturated the remaining three TF positions step-by-step still with an increment of $\Delta \Theta=0.25$, resulting in an overall coverage of $\Theta=4.0$ with an $E_{a d, Z P E, H_{n}}=-5.30 \mathrm{eV}$ (Table 4).

Energetically, the $E_{a d}^{n+1}$ trend beyond $\Theta=2.0$ on the $\mathrm{W}(100)$ surface is quite interesting. Adding one hydrogen atom to the surface $(\Theta=2.25)$ is essentially energetically nonconsequential with an $E_{a d}^{n+1}$ of $0.01 \mathrm{eV}$ corrected from the ZPE, however at $\Theta=2.5$ and $\Theta=2.75$ the $E_{a d}^{n+1}$ values are $0.16 \mathrm{eV}$ and $0.24 \mathrm{eV}$ and the global trend is positive. Thus there is no stabilization as more molecular hydrogen adsorbs, indicating the adsorption of further hydrogen is energetically non-favorable. Once $\Theta=3.0$ is reached, corresponding to full occupation of TF positions, the $E_{a d}^{n+1}$ to is $-0.05 \mathrm{eV}$. Again, at $\Theta=3.25$ and 3.5 the $E_{a d}^{n+1}$ values are negative $-0.08 \mathrm{eV}$ and $-0.09 \mathrm{eV}$ but nevertheless not significant. This 
corresponds to the addition of two T position hydrogen atoms.

Upon the addition of another hydrogen to the $\mathrm{W}(100)$ surface at $\Theta=4.0$, coverage $\Theta=4.25$ is reached. The only site we were able to make converge was the top site T shown in Table 4 with an $E_{a d, Z P E, H_{n}}=-4.80 \mathrm{eV}$. In that configuration, a pre-dissociated $\mathrm{H}_{2}$ molecule is formed that sits atop a $\mathrm{W}$ atom. The two hydrogen atoms are separated by just $0.83 \AA$ (close to the molecular $\mathrm{H}_{2}$ bond length of $\approx 0.75 \AA$ ) and $1.9 \AA$ above the surface plane, a bit higher than the other hydrogens at $\mathrm{T}$ positions. This configuration was revealed to be unstable; indeed, it leads to desorption of a hydrogen molecule with a stabilization in energy of 0.49 eV. A Nudge Elastic Band (NEB) calculation shows that from this pre-dissociation state, a $\mathrm{H}_{2}$ molecule can desorb from the surface with zero activation energy.

For $E_{a d, H_{n}}$ and $E_{a d, Z P E, H_{n}}$ of all intermediary coverage ratios not presented in Tables $3 \& 4$ please see the supplementary information.

\section{Saturation of the surfaces and impact on hydrogen desorption measurements}

Figure 3 presents plots of the $E_{a d, Z P E, H_{n}}$ vs. the hydrogen to tungsten coverage ratio $(\Theta)$ for the most stable configurations we investigated for the W (110) and W (100) systems. In the case of the $\mathrm{W}(110)$ surface a clear minimum exists near coverage $\Theta=1.0$. Thermodynamically, coverage ratios around 1.0 hydrogen atom to surface tungsten atom should be favored. The corresponding minimum in which there is a TF position hydrogen corresponding to each tungsten can be seen in Table 1. This represents a mono-layer $(\theta=$ 1.0) configuration in which hydrogens lay $1.1 \AA$ above the surface and exhibit the same symmetry as the underlying tungsten atoms but translated by $0.5 \boldsymbol{a}$ and $0.7 \boldsymbol{b}$ in the [001] and[1ํㅣㅇ directions, respectively. 
For the $\mathrm{W}(100)$ surface, there is a minimum at $\Theta=2.0$, precisely where all SB positions are occupied and the known surface reconstruction of the bare W(100) is fully removed. This also corresponds to one monolayer $(\theta=1.0)$ of hydrogen on top of the surface. After $\Theta=2.0$, the $E_{a d, Z P E, H_{n}}$ rises slowly so one would expect that a coverage around $\Theta=2.0$ is optimal. Though the coverage is doubled for the W(100) surface, again the favored configuration represents a mono-layer of hydrogen at nearly the same height as the $\mathrm{W}(110)$ surface.

During TDS experiments, the base pressure is very low, and the hydrogen coverage ratio of the surface is expected to be very low. Looking at our results at low energy coverage ratio; the binding energies are in the range of $-0.7 \mathrm{eV}$ to $-0.86 \mathrm{eV}$ at coverage 0.5 for the $\mathrm{W}(110)$ and $\mathrm{W}(100)$ surfaces, respectively. This is the binding energy of a hydrogen atom in a tungsten vacancy filled with 5 hydrogen atoms ${ }^{28}$. Consequently, we can conclude that the effect of the surface on hydrogen retention and desorption from the surface cannot be neglected for the interpretation of TDS measurements.

\section{Comparison with low energy ion beam experiments}

Both LEIS and DRS use low energy ion beams (typically $\mathrm{He}^{+}, \mathrm{Li}^{+}$, or $\mathrm{Ne}^{+}$) to probe the surface and are capable of directly detecting the atomic binding configuration of chemisorbed H. Consequently, they were used on the hydrogenated $\mathrm{W}(100)$ and $\mathrm{W}(110)$ surfaces and complement the results of our DFT study. Surface composition is derived from the energies of the scattered and recoiled particles, whereas the local atomic structure can be determined from shadowing effects between adjacent surface atoms. To gain further sensitivity to the chemisorbed hydrogen, we recently developed an approach that involves aligning the analysis beam so that it impinges on the surface at a grazing angle ${ }^{10}$. Under such conditions, the incident ions, rather than undergoing discrete "binary" collisions with surface atoms, instead 
are deflected along open surface channels. An observation of recoiled hydrogen along one of surface channel. This information can be used to determine the positioning of the $\mathrm{H}$ relative to the neighboring $\mathrm{W}$ atoms, and therefore enables us to uniquely identify the binding site. More detailed information, such as the H-W bond length may be determined by simulating the scattering process using molecular dynamics and comparing this information quantitatively with experiment. LEIS and DRS therefore offer direct structural information that complements prior studies using temperature-programmed desorption and vibrational spectroscopies, and can be used as a basis for comparison with the atomic detail of DFT results.

Previously we used both LEIS and DRS to analyze the $\mathrm{W}(100)+\mathrm{H}$ adsorption system, as described in Refs. [10] and [11]. Using a $1 \mathrm{keV} \mathrm{Ne}^{+}$analysis beam, we mapped the recoiled hydrogen flux at varying incidence angles $(\alpha)$ and azimuthal rotations of the crystal $(\phi)$. A subset of these data, along with angle definitions, appears in Fig. 4 at a representative fixed angle of incidence $\left(\alpha=81^{\circ}\right)$ for both (a) a saturation coverage $(\Theta=2.0)$ of chemisorbed $\mathrm{H}$ and (b) a partial coverage $(\Theta \approx 0.25)$. We found that dosing the surface with molecular $\mathrm{H}_{2}$ at a partial pressure of $3 \times 10^{-7}$ Torr was sufficient to reach a saturation at $25^{\circ} \mathrm{C}$. (Alternatively, varying the sample temperature up to $250{ }^{\circ} \mathrm{C}$ while dosing the surface could be used as a means to more precisely control the coverage.) In all cases we observed enhanced $\mathrm{H}$ recoil signals along $\langle 100\rangle$ and $\langle 110\rangle$ surface channels, consistent with H occupying SB sites. To confirm this finding, we simulated a subset of the scattering data for a saturation $\mathrm{H}$ coverage using molecular dynamics. These simulations reproduced the experiments well when hydrogen was placed in bridge sites at a height of $1.0-1.2 \AA$ above the surface. Both of these findings are very much consistent with the DFT calculations discussed earlier in this article. 
It is worthwhile considering how changes in atomic positions may affect ion channeling along the surface, and ultimately the azimuthal variation in the recoiled hydrogen flux at different coverages. As previously mentioned, maxima in the recoiled flux were observed along the $\langle 100\rangle$ and $\langle 110\rangle$ azimuths. However, the width of these maxima varied as a function of coverage. When the surface was saturated with hydrogen, the observed recoil patterns subtended a FWHM of $\pm 16^{\circ}$ along the $\langle 100\rangle$ directions, as indicated by the dashed lines in Fig. 4(a). The width of these features decreased to $\pm 10^{\circ}$ when only a residual coverage $(\Theta \approx 0.25)$ was present. We observed a similar decrease along the $\langle 110\rangle$ directions, from $\pm 10^{\circ}$ at saturation to $\pm 8^{\circ}$ at partial coverage.

Our DFT simulations predict a $(\sqrt{2} \times \sqrt{2}) R 45$ reconstruction of the $\mathrm{W}(100)$ surface at partial $\mathrm{H}$ coverage, where the first-layer tungsten atoms deflect laterally toward hydrogen adatoms residing in short bridge sites. While these defections are small, they nevertheless narrow the widths of the surface channels by approximately $0.4 \AA$ along the $\langle 100\rangle$ directions. This reduces the degree of misalignment that is tolerable for channeling of the incident $\mathrm{Ne}^{+}$ions. Assuming the recoiled $\mathrm{H}$ resides within short bridge sites, this would have a commensurate effect on the recoiled $\mathrm{H}$ features. The data interpretation is complicated somewhat by the details of how the incident $\mathrm{Ne}$ are deflected by the chemisorbed $\mathrm{H}$ layer. Local minima (indicated by the markers "a" and "b" within Fig. 4) are due to blocking by the presence of an ordered layer of chemisorbed $\mathrm{H}$ along the surface channels. The number of $\mathrm{H}$ adatoms within a particular surface channel, as well as their height would be expected to also contribute to this effect. Setting this consideration aside, however, our results are certainly consistent with the reconstruction and preferred hydrogen binding site predicted by the DFT simulations. 
These findings motivate consideration of the $\mathrm{W}(110)+\mathrm{H}(\mathrm{ads})$ system, which in many respects is "simpler" to investigate from an experimental standpoint than the W(100) atomic plane. For example, our DFT calculations predict that upon adsorption of hydrogen, reconstruction of the first layer W(110) is rather modest, involving only a lateral shift of the first layer relative to the second, as discussed in Sect. 3.1. Furthermore, DFT predicts that the height of the hydrogen layer above the surface does not vary with coverage. With these simplifications, the detailed interpretation of the recoiled hydrogen is much more straightforward. We can then directly take into account the interaction of the incident $\mathrm{Ne}^{+}$with the adsorbed hydrogen layer without having to consider the additional complication of structural changes.

Initial results from this work are illustrated in Fig. 5, showing the azimuthal variation in recoiled hydrogen flux for the $\mathrm{W}(110)+\mathrm{H}(\mathrm{ads})$ system. Here we have used experimental procedures nearly identical to our previous work described in Ref. [10]. As before, we used a $1 \mathrm{keV} \mathrm{Ne}{ }^{+}$analysis beam to probe the chemisorbed hydrogen structure, and collected the scattered and recoiled particles using an electrostatic analyzer positioned at a $45^{\circ}$ angle relative to the incident ion beam. We noted that adsorption of hydrogen did not affect the positioning of the scattering peaks from the W(110) substrate. This is consistent with the first atomic layer of the surface remaining in a $1 \times 1$ configuration, as predicted by our DFT calculations. The data depicted in Fig. 5 illustrates a single azimuthal scan at a fixed incidence angle of $\alpha=81^{\circ}$. To aid in the interpretation of these data, a schematic of the corresponding orientation of the W(110) substrate appears to the right. Note that in Fig. 5, the [001] surface direction corresponds to an azimuthal angle of $\phi=90^{\circ}$.

We can use the information contained in Fig. 5 to perform a preliminary identification of the 
preferred hydrogen binding site on the W(110) surface. Let us first consider the possibility within any surface channels. This configuration would not produce any recoiled hydrogen variation with azimuth, which is clearly not in agreement with our experiments. Key pieces of evidence from Fig. 5 are the presence of increased recoiled hydrogen intensity along the $\langle 001\rangle$ surface channels, but not along $\langle 1 \overline{1} 0\rangle$ directions. Long bridge sites are not present in the troughs between the $\mathrm{W}$ surface atoms along the $\langle 001\rangle$ azimuths, and can therefore be eliminated from consideration. On the other hand, occupation of short bridge sites would result in the presence of hydrogen along the $\langle 1 \overline{1} 0\rangle$ surface channels. However, from the azimuthal scan depicted in Fig. 5, no enhanced recoil yield along these directions is present that would be consistent with SB binding. Of the possible configurations of $\mathrm{H}$ on the $\mathrm{W}(110)$ surface considered with our DFT analysis, only occupation of three-fold sites would produce a recoiled hydrogen pattern consistent with our experiments (i.e., enhanced hydrogen recoil intensity observed along $\langle 001\rangle$ azimuths but not along the $\langle 1 \overline{1} 0\rangle$ directions.) This result is in agreement with our DFT calculations. A more comprehensive analysis of the chemisorbed hydrogen (including complete maps of the recoiled hydrogen fluxes at varying coverage) will be presented in a forthcoming publication.

\section{Conclusion}

We investigated the saturation limits of hydrogen on the (110) and (100) surfaces of tungsten via Density Functional Theory (DFT) by studying stable configurations at low coverage ratios up to the point beyond experimental saturation. Our findings lead us to the general prediction that saturation exists on each surface when one monolayer of hydrogen is adsorbed $\approx 1.0 \AA$ above the surface plane. In the case of (110) this exists at a hydrogen to surface tungsten coverage ratio of 1.0, while on the (100) surface the coverage ratio is 2.0. In both cases, this 
corresponds to one monolayer of hydrogen on the surface.

The DFT results we computed were also compared with experimental Low Energy Ion Scattering and Direct Recoil Spectroscopy measurements. These measurements agree with the following findings for the $\mathrm{W}(100)$ surface: (i) a strong $(\sqrt{2} \times \sqrt{2}) R 45$ reconstruction of the is observed at low coverage and tends to vanish for one monolayer of $\mathrm{H}$ atoms, (ii) when saturation is reached the surface reconstruction is lost, (iii) saturation corresponds to a monolayer of adsorbed $\mathrm{H}$ atoms occupying all the Short-Bridge positions and a coverage of 2.0 hydrogen atoms with respect to the number of $\mathrm{W}$ surface atoms.

Similarly, experiments confirm the following findings on the W(110) surface: (i) the first layer $\mathrm{W}$ atoms remain in a $(1 \times 1)$ configuration upon chemisorption of $\mathrm{H}$, (ii) the Three-Fold positions are preferred at all coverage investigated experimentally.

In the end, this work demonstrates that the effect of the surface cannot be neglected when trying to understand the retention and desorption properties for hydrogen with tungsten, and that the surface can act as a sink for hydrogen at coverage ratios below one monolayer.

\section{Acknowledgment}

This work has been carried out within the framework of the EUROfusion Consortium and has received funding from the Euratom research and training programme 2014-2018 under grant agreement No 633053 and also the A*MIDEX project ( $\mathrm{n}^{\circ}$ ANR-11-IDEX-0001-02) funded by the 'Investissements d'Avenir' French Government program, managed by the French National Research agency (ANR). The views and opinions expressed herein do not necessarily reflect those of the European Commission. The authors of this work were granted access to the HPC resources of IDRIS and CINES under the allocation A0020806612 made 
by GENCI (Grand Equipement National de Calcul Intensif) and to the Marconi Supercomputer at CINECA SuperComputing Application and Innovation Department, Bologna, Italy.

\section{Bibliography}

${ }^{1}$ G. Federici, Plasma wall interaction in ITER, Phys. Scr. T124 (2006) 1-8

${ }^{2}$ S. Brezinsek and JET-EFDA contributors , Plasma-surface-interaction in the Be/W environment: Conclusions drawn from the JET-ILW for ITER, Journal of Nuclear Materials 463 (2015) 11-21

${ }^{3}$ T. Rayment, R. Sclögl, J. M. Thomas and G. Ertl. Structure of the ammonia synthesis catalyst, Nature 315 (1985) 311

${ }^{4}$ G Ertl and H. J. Freund, Catalysis and surface sciencePhysics today, 52 (1999) 32

${ }^{5}$ T. E. Felter, R. A. Barker, and P. J. Estrup, Phase transition on Mo(100) and W(100) surfaces, Phys. Rev. Lett. 38 (1977) 1138.

${ }^{6}$ M. K. Debe and D. A. King, Phase group determination of the low-temperature W(100) $(\sqrt{2} \times \sqrt{2})$ R45 surface by low-energy-electron diffraction, Phys. Rev. Lett. 39 (1977) 708

${ }^{7}$ R. A. Barker, and P. J. Estrup, Hydrogen on tungsten (100) : adsorbate-induced surface reconstruction, Phys. Rev. Lett. 41 (1978) 1307

${ }^{8}$ I. Stensgaard, L. C. Feldman, and P. J. Silverman, Reconstruction of the W(001) surface and its reordering by hydrogen addsorption, studied by MeV ion scattering, Phys. Rev. Lett. 42 (1979) 247

${ }^{9}$ M. R. Barnes and R. F. Willis, Hydrogen-Adsorption-Induced Reconstruction of Tungsten (100): Observation of Surface Vibrational Modes, Phys. Rev. Lett. 41 (1978) 1729. 
${ }^{10}$ R. D. Kolasinski, N. C. Bartelt, J. A. Whaley, and T. E. Felter, Channeling of low-energy ions on hydrogen-covered single-crystal surfaces, Phys. Rev. B 85 (2012) 115422.

${ }^{11}$ R. D. Kolasinski, K. D. Hammond, J. A. Whaley, D. A. Buchenauer, B. D. Wirth, Analysis of hydrogen adsorption and surface binding configuration on tungsten using direct recoil spectrometry, J. Nucl. Mater 463 (2015) 1053-1056.

${ }^{12}$ L. D. Roelofs and D. J. Estrup, Two-dimensional phases in chemisorption system, Surf. Sci 125 (1983) 51.

${ }^{13}$ G. B. Blanchet, N. J. Dinardo, and E. W. Plummer, H on tungsten (110) studied by angle resolved photoemission and inelastic electron scattering, Surf. Sci 118 (1982) 146

${ }^{14}$ T. U. Nahm, and R. Gomer, The adsorption of hydrogen on W(110) and Fe covered W(110) surfaces, Surf. Sci. 375 (1997) 281-292

${ }^{15}$ M. Balden, S. Lehwald, and H. Ibach, Substrate and hydrogen phonons of the ordered $\mathrm{p}(2 \times 1)$ and $(2 \times 2)$ phase and of the anomalous $(1 \times 1)$ phase on hydrogen on $\mathrm{W}(110)$, Phys Rev B 53 (1996) 7479.

${ }^{16}$ M. Balden, S. Lehwald, H. Ibach, and D. L. Mills, Hydrogen covered W(110) surface: a hydrogen liquid with a propensity for one dimensional order, Phys Rev. Lett. 73 (1994) 854.

${ }^{17}$ E. Hulpke, and J. Lüdecke, Hydrogen-induced phonon anomaly on W(110) surface, Phys. Rev. Lett. 68 (1992) 2846

${ }^{18}$ J. W. Chung, S. C. Ying, and P. J. Estrup, Reconstruction of the W(110) surface induced by hydrogen adsorption, Phys. Rev. Lett. 56 (1986) 749.

${ }^{19}$ K. Heinola and T. Ahlgren, First principle study of $\mathrm{H}$ on the reconstructed W(100) surface, Phys. Rev. B 81, (2010) 073409

${ }^{20}$ D. F. Jhonson and E. A. Carter, Hydrogen in tungsten: absorption, diffusion, vacancy trapping, and decohesion, J. Mater. Res. 25 (2010) 315

${ }^{21}$ A. Moitra, K. Solanki, Adsorption and penetration of hydrogen in W: a first principles 
study Computational Materials Science 50 (2011) 2291-2294

${ }^{22}$ A. Nojima, and K. Yamashita, A theoretical study of hydrogen adsorption and diffusion on W(110) surface, Surf. Sci. 601 (2007) 3003.

${ }^{23}$ L. Sun, S. Jin, G. H. Lu, L. Wang, High hydrogen retention in the sub-surfaces of tungsten plasma facing materials: A theoretical insight, Scripta Materialia 122 (2016) 14-17

${ }^{24}$ C. L. Guerrero, N. Gordillo, R. Iglesias, J. M. Perlado and C. Gonzalez, Ab initio study of tungsten defects near the surface, Modelling Simul. Mater. Sci. Eng. 24 (2016) 045006

${ }^{25}$ H. F. Busnengo, and A. E. Martinez, $\mathrm{H}_{2}$ chemisorption on W(100) and W(110) surfaces, J. Phys. Chem. C 112 (2008) 5579

${ }^{26}$ R. Pétuya, P. Larrégaray, C. Crespos, H. F. Busnengo and A. E. Martínez, Revisiting the nonreactive scattering of $\mathrm{N}_{2}$ off $\mathrm{W}(100)$ : on the influence of the scattering azimuth on inplane angular distributions, J. Chem. Phys. 141 (2014) 0242701

${ }^{27}$ R. Pétuya, C. Crespos, E. Quintas-Sanchez and P. Larrégaray, Comparative theoretical study of $\mathrm{H}_{2}$ Eley-Rideal recombination dynamics on W(100) and W(110)J. Phys. Chem. C 118 (2014) 11704-11710

${ }^{28}$ N. Fernandez, Y. Ferro and D. Kato, Hydrogen diffusion and vacancies formation in tungsten: Density Functional Theory calculations and statistical models, Acta Materialia 94 (2015) 307-318

${ }^{29}$ K. Ohsawa, F. Nakamori, Y. Hatano, M Yamaguchi, Thermodynamics of hydrogeninduced superabundant vacancy in tungsten, J. of Nucl. Mater. 458 (2015) 187-197

${ }^{30}$ L. Sun, S. Jin, H. Zhou, Y. Zhang, W. Zhang, Y. Ueda, H. T. Lee and G. Lu, Critical concentration of hydrogen bubble formation in metals, J. Phys : Cond Mater 26 (2014) 395402

31 T. Oda, Thermodynamic model for grain boundary effects on hydrogen solubility, diffusivity and permeability in poly-crystalline tungsten, Fusion Engineering and Design 112 
(2016) 102-116.

${ }^{32}$ E. A. Hodille, X. Bonnin, R. Bisson, T. Angot, C.S. Becquart, J. M. Layet, C. Grisolia, Macroscopic rate equation modeling of trapping/detrapping of hydrogen isotopes in tungsten materials, J. Nucl. Mater 467 (2015) $424-431$

${ }^{33}$ E. A. Hodille, Y. Ferro, N. Fernandez, C. S. Becquart, T. Angot, J.-M. Layet, R. Bisson, C. Grisolia, Study of hydrogen isotopes behavior in tungsten by a multi trapping macroscopic rate equation model, Phys. Scrip. T167 (2016) 014011

${ }^{34}$ K. Schmid, U. von Toussaint, T. Schwarz-Selinger, Transport of hydrogen in metals with occupancy dependent trap energies, J. Appl. Phys. 116 (2014) 134901

35 T. Ahlgren, K. Heinola, K. Vörtler, J. Keinonen, Simulation of irradiation induced deuterium trapping in tungsten, J. Nucl. Mater. 427 (2012) 152-161

36 J. Guterl, R. D. Smirnov, S. I. Krasheninnikov, M. Zibrov, A. A. Pisarev, Theoretical analysis of deuterium retention in tungsten plasma-facing components induced by various traps via thermal desorption spectroscopy, Nucl. Fusion 55 (2015) 093017

37 O. V. Ogorodnikova, J. Roth, M. Mayer, Ion-driven deuterium retention in tungsten, J. Appl. Phys., 103 (2008) 034902

${ }^{38}$ M. Zibrov, S. Ryabstev, Y. Gasparyan, A. Pisarev, Experimental determination of the deuterium binding energy with vacancies in tungsten, J. Nucl. Mater. 477 (2016) 292-297 ${ }^{39}$ A. D. Quastel, J. W. Davis, A. A. Haasz, R. G. Macaulay-Newcombe, Effect of post-D ${ }^{+}-$ irradiation time delay and pre-TDS heating on D retention in single crystal tungsten, J. Nucl. Mater. 359 (2006) 8

40 A.A. Haasz, J.W. Davis, M. Poon, R. G. Macaulay-Newcombe, Deuterium retention in tungsten for fusion use, J. Nucl. Mater. 258-263 (1998) 889

41 J. Roszell, J.W. Davis, A.A. Haasz, Temperature dependence of deuterium retention mechanisms in tungsten, J. Nucl. Mater. 429 (2012) 48 
${ }^{42}$ M. Poon, A.A. Haasz, J.W. Davis, R. G. Macaulay-Newcombe, Impurity effects and temperature dependence of D retention in single crystal tungsten, J. Nucl. Mater. 313-316 (2003) 199

${ }^{43}$ R. Bisson, S. Markelj, O. Mourey, F. Ghiorghiu, K. Achkasov, J.-M. Layet, P. Roubin, G. Cartry, C. Grisolia, T. Angot, Dynamic fuel retention in tokamak wall materials: An in situ laboratory study of deuterium release from polycrystalline tungsten at room temperature, J. Nucl. 467 (2015) 432-438

${ }^{44}$ A. Manhard, K. Schmid, M. Balden, W. Jacob, Influence of the microstructure on the deuterium retention in tungsten, J. Nucl. Mater. 415 (2011) S632-S635

45 J. P. Perdew, K.Burke, and M.Ernzerhof, Generalized gradient approximation made simple, Phys. Rev. Lett, 77 (1996) 3865-3868

46 D.Vanderbilt, Soft self-consistent pseudopotentials in generalized eigenvalue formalism, Phys. Rev. B, 41 (1990) 7892-7895

${ }^{47}$ S. Baroni, S. de Gironcoli, A. Dal Corso, Phonons and related crystal properties from density-functional perturbation theory, Rev. Mod. Phys. 73 (2001) 515

${ }^{48}$ P. Giannozzi, S. Baroni, N. Bonini, M. Calandra, R. Car, C. Cavazzoni, D. Ceresoli, G. L. Chiarotti, M. Cococcioni, I Dabo, A. Dal Corso, S. Fabris, G. Fratesi, S. de Gironcoli, R. Gebauer, U. Gerstmann, Ch. Gougoussis, A. Kokalj, M. Lazzeri, L. Martin-Samos, N. Marzari, F. Mauri, R. Mazzarello, S. Paolini, A. Pasquarello, L. Paulatto, C . Sbraccia, S. Scandolo, G. Sclauzero, A. P. Seitsonen, A. Smogunov, P. Umari, R.M. Wentzcovitch, QUANTUM ESPRESSO: a modular and open-source software project for quantum simulations of materials, J. Phys.: Condens. Mater 21 (2009) 395502

${ }^{49}$ P. Ferrin, S. Kandoi, A. U. Nilekar, and M. Mavrikakis, Hydrogen adsorption, absorption and diffusion on and in transition metal surfaces: A DFT study, Surface Science 606 (2012) $679-689$ 


\section{Figure 1}

A

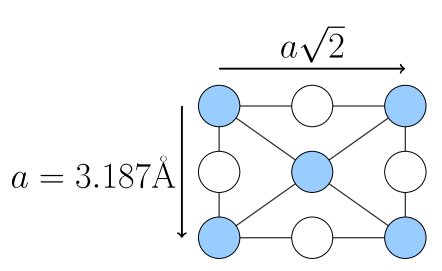

B

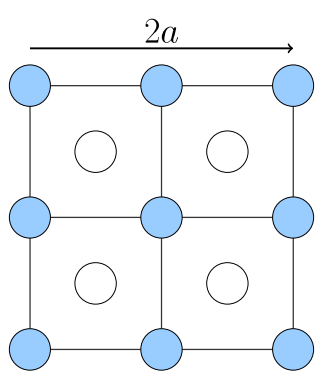

C

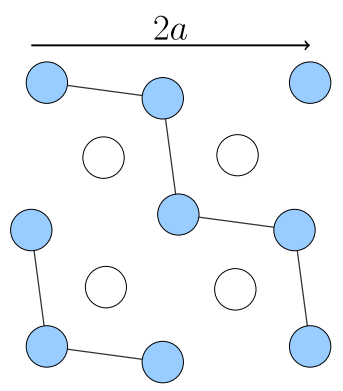

Fig. 1. Top-view representations of the (A) W(110), (B) W(100), and (C) reconstructed W(100) surface definitions used in this work. Light blue circles $\bigcirc$ represent surface tungsten atoms. White circles $\bigcirc$ represent sub-surface tungsten atoms one layer below the surface. 


\section{Figure 2}

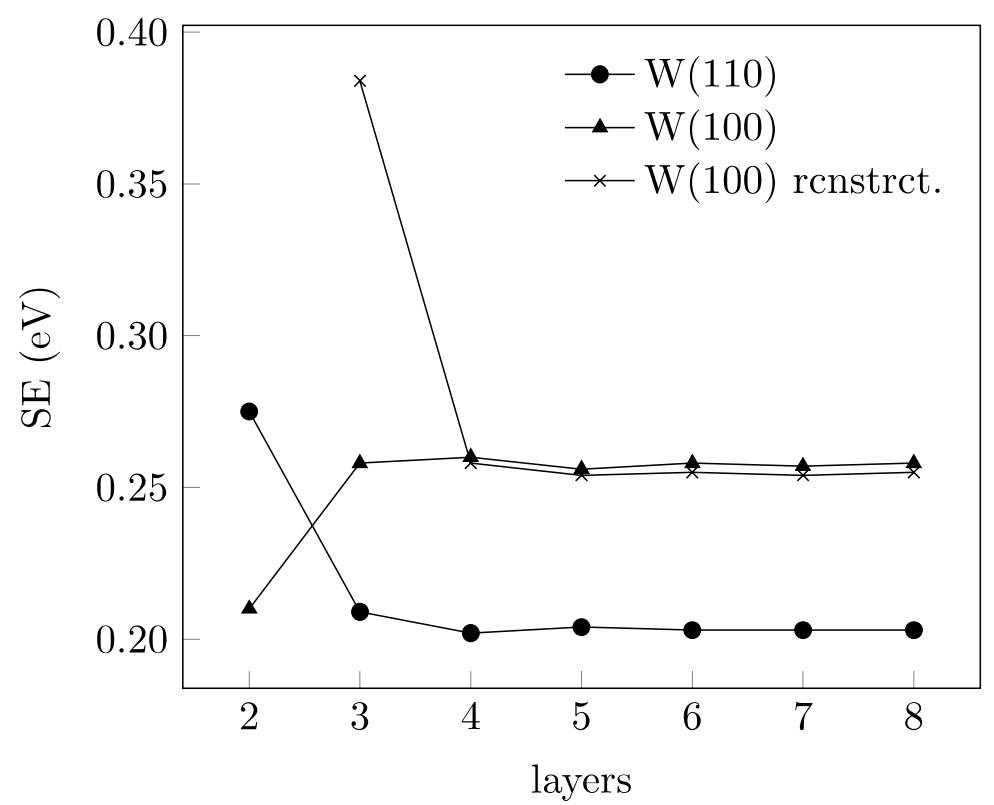

Fig. 2. Calculated surface energies (SE) of the $\mathrm{W}(110), \mathrm{W}(100)$, and reconstructed W(100) surfaces vs. the number of layers in the slab model. 


\section{Figure 3}

A
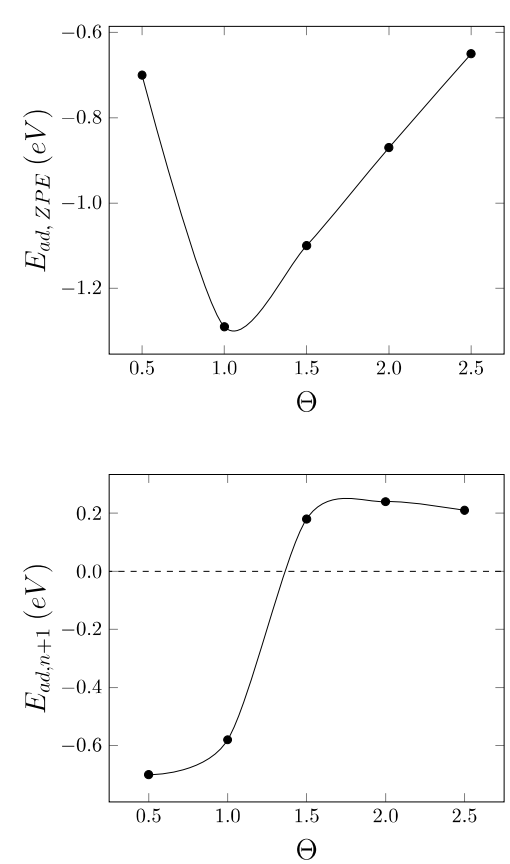

B
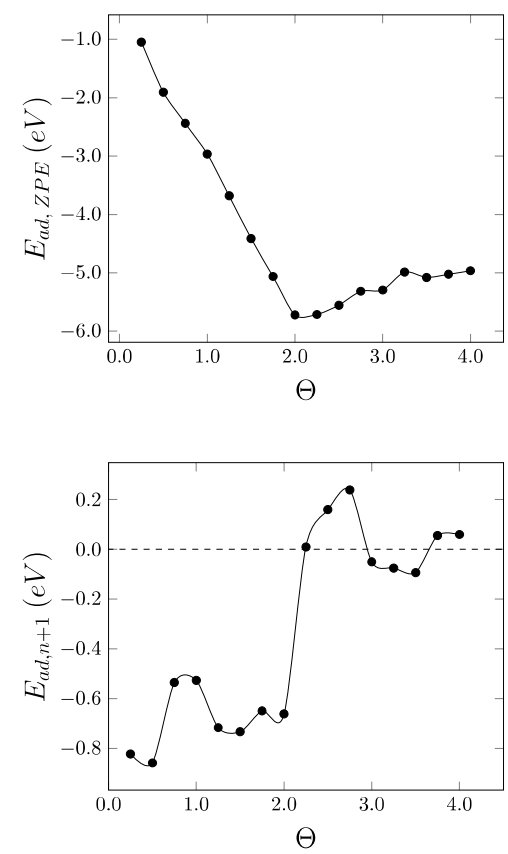

Fig. 3. Plots of the lowest energy $E_{a d, Z P E}$ and $E_{a d, n+1}$ vs. hydrogen coverage ratio $(\Theta)$ of the $\mathbf{A} \mathrm{W}(110)$ and $\mathbf{B} \mathrm{W}(100)$ surfaces. 


\section{Figure 4}

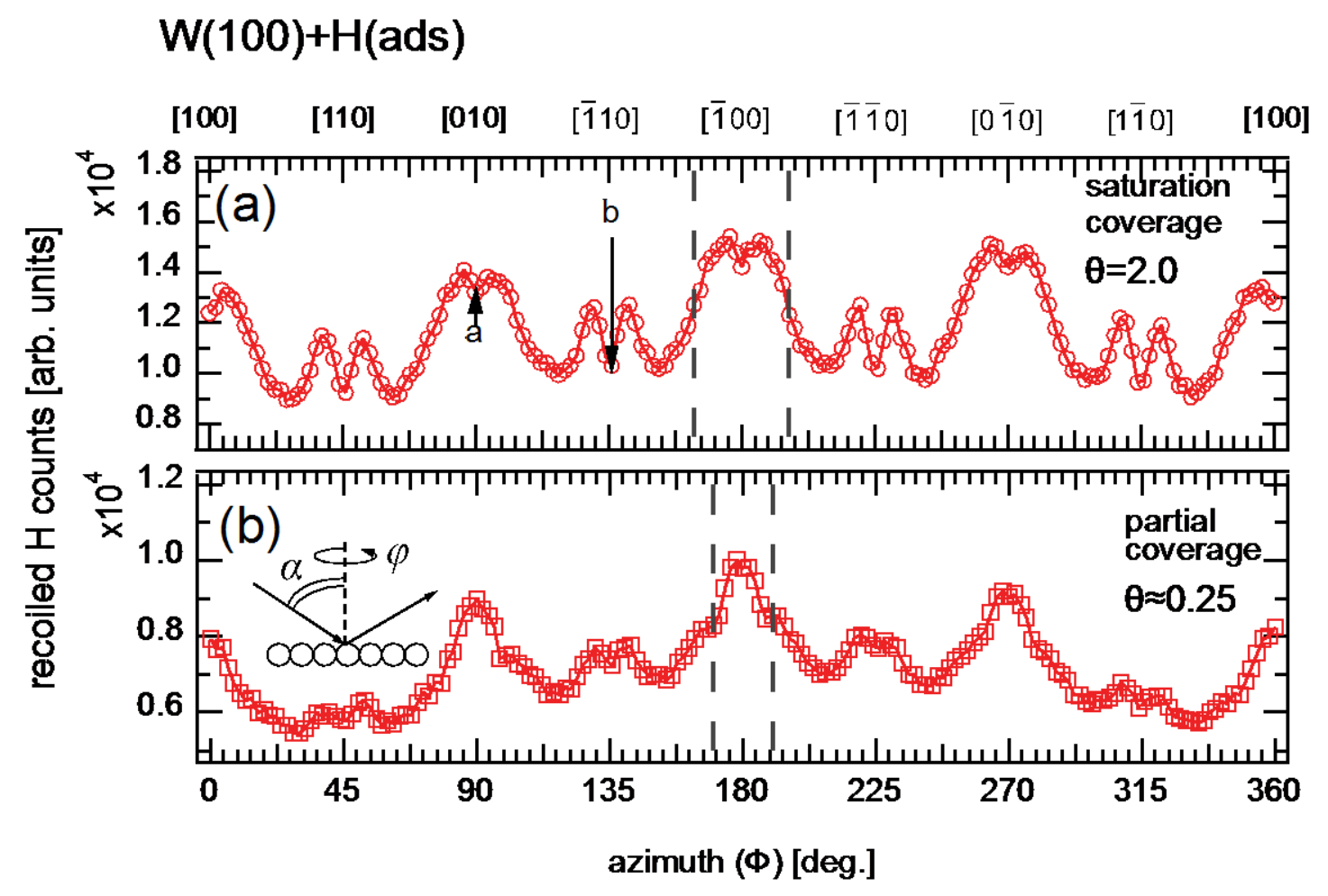

Fig. 4: Azimuthal scans showing the variation in hydrogen recoil intensity as a function of azimuth for $1 \mathrm{keV} \mathrm{Ne} \rightarrow \mathrm{W}(100)+\mathrm{H}($ ads). The inset shows the definitions of the incidence angle $(\alpha)$ and crystal azimuth $(\phi)$. The above measurements were acquired at a fixed $\alpha=81^{\circ}$ and a scattering angle of $45^{\circ}$ relative to the incident beam. 


\section{Figure 5}

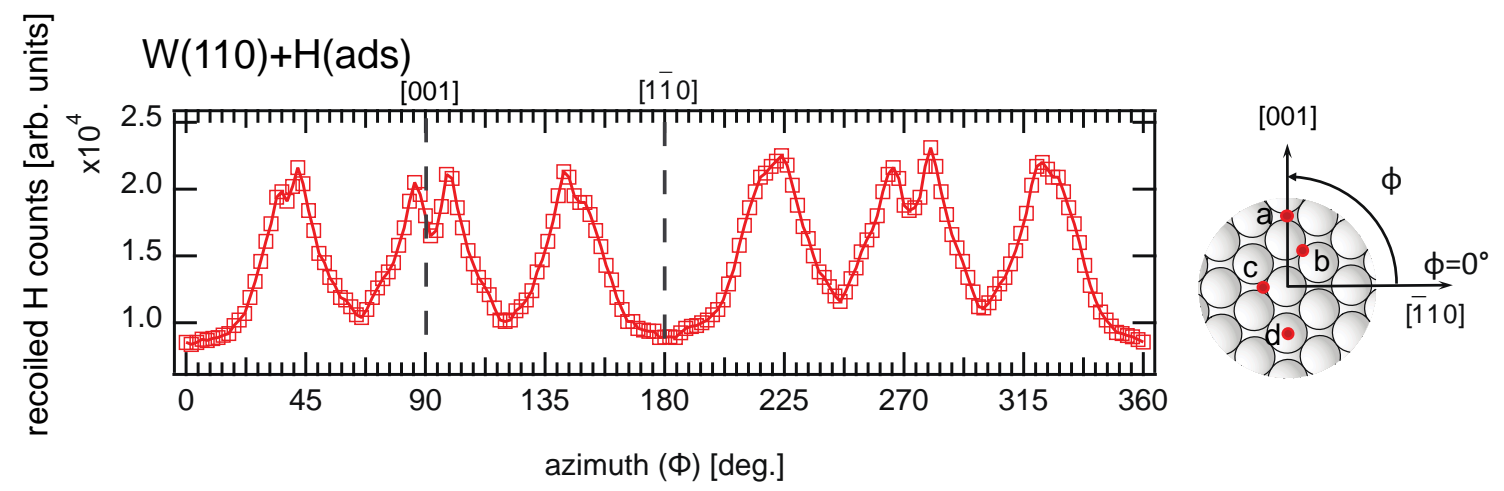

Fig. 5: Azimuthal scan at fixed incidence $\left(\alpha=81^{\circ}\right)$ and a scattering angle of $45^{\circ}$ relative to the incident beam $(1 \mathrm{keV} \mathrm{Ne} \rightarrow \mathrm{W}(100)+\mathrm{H}(\mathrm{ads})$.) Inset: the orientation of the $\mathrm{W}(110)$ substrate, indicating the locations of possible $\mathrm{H}$ chemisorption sites, including (a) long bridge (LB), (b) short bridge (SB), (c) three-fold (TF), and (d) top (T) sites. 


\section{Table 1}

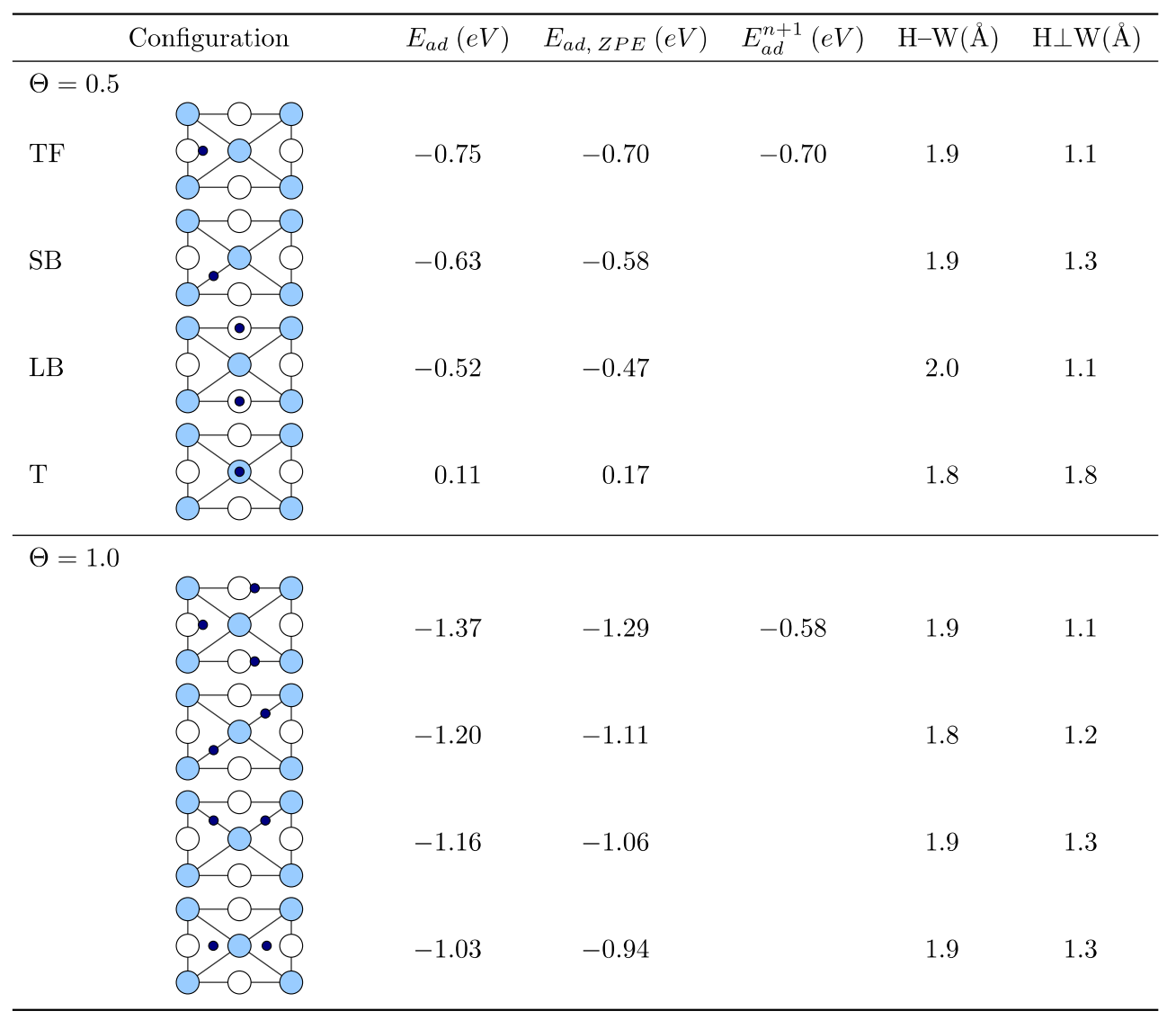

Table 1. Top-view configurational representations, energetic data, and characteristic geometric data at $\Theta=0.5 \& 1.0$ for the most stable configurations on the $\mathrm{W}(110)$ surface. $\mathrm{H}-\mathrm{W}$ distances reported are the distance of hydrodrogen to the nearest tungsten atom while $\mathrm{H} \perp \mathrm{W}$ is the distance of hydrogen above the surface plane. 
Table 2

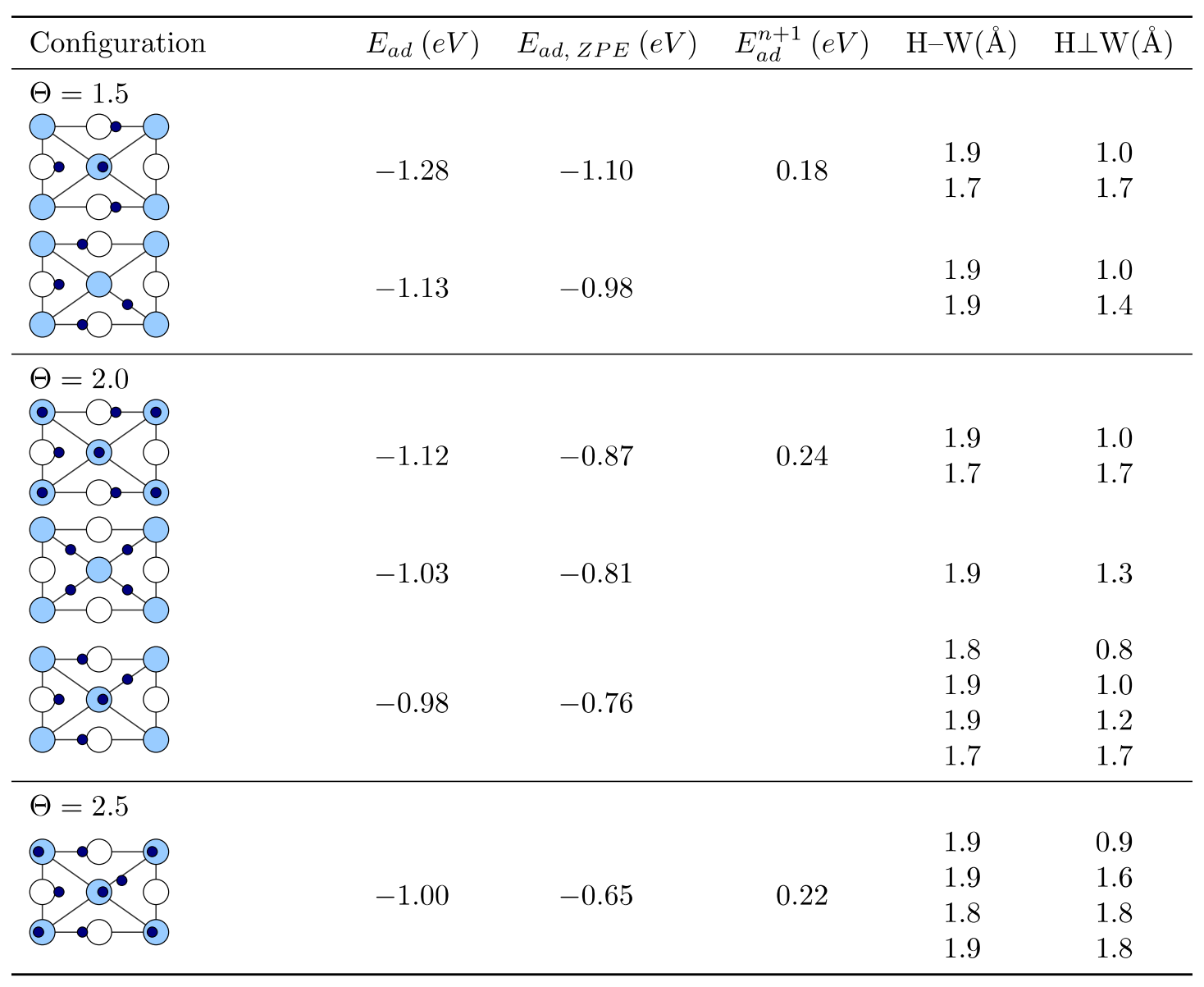

Table 2. Top-view configurational representations, energetic data, and characteristic geometric data at $\Theta=1.5,2.0, \& 2.5$ for the most stable configurations on the $\mathrm{W}(110)$ surface. $\mathrm{H}-\mathrm{W}$ distances reported are the distance of hydrodrogen to the nearest tungsten atom while $\mathrm{H} \perp \mathrm{W}$ is the distance of hydrogen above the surface plane. 
Table 3

\begin{tabular}{llllll}
\hline Configuration & $E_{a d}(\mathrm{eV})$ & $E_{a d, Z P E}(\mathrm{eV})$ & $E_{a d}^{n+1}(\mathrm{eV})$ & $\mathrm{H}-\mathrm{W}(\AA)$ & $\mathrm{H} \perp \mathrm{W}(\AA)$ \\
\hline & & & & & \\
& & & & \\
\end{tabular}

Table 3. Top-view configurational representations, energetic data, and characteristic geometric data at $\Theta=0.5,1.0,1.5, \& 2.0$ for the most stable configurations on the $\mathrm{W}(100)$ surface. $\mathrm{H}-\mathrm{W}$ distances reported are the distance of hydrodrogen to the nearest tungsten atom while $\mathrm{H} \perp \mathrm{W}$ is the distance of hydrogen above the surface plane. 


\section{Table 4}

\begin{tabular}{|c|c|c|c|c|c|}
\hline Configuration & $E_{a d}(\mathrm{eV})$ & $E_{a d, Z P E}(e V)$ & $E_{a d}^{n+1}(e V)$ & $\mathrm{H}-\mathrm{W}(\AA)$ & $\mathrm{H} \perp \mathrm{W}(\AA)$ \\
\hline$\Theta=3.0$ & -5.82 & -5.07 & -0.05 & $\begin{array}{l}1.9 \\
1.8\end{array}$ & $\begin{array}{l}1.1 \\
0.1\end{array}$ \\
\hline 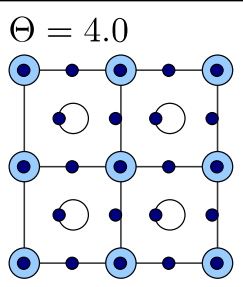 & -5.82 & -4.47 & 0.06 & $\begin{array}{l}1.9 \\
1.8 \\
1.7\end{array}$ & $\begin{array}{c}1.0 \\
-0.1 \\
1.7\end{array}$ \\
\hline 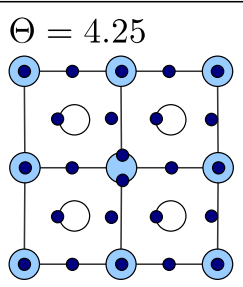 & -5.79 & -4.80 & -0.06 & $\begin{array}{l}1.8 \\
1.8 \\
1.7 \\
1.9\end{array}$ & $\begin{array}{c}1.0 \\
-0.1 \\
1.7 \\
1.9\end{array}$ \\
\hline
\end{tabular}

Table 4. Top-view configurational representations, energetic data, and characteristic geometric data at $\Theta=3.0,4.0 \& 4.25$ for the most stable configurations on the $\mathrm{W}(100)$ surface. $\mathrm{H}-\mathrm{W}$ distances reported are the distance of hydrodrogen to the nearest tungsten atom while $\mathrm{H} \perp \mathrm{W}$ is the distance of hydrogen above the surface plane. 


\section{Supplementary Material
Click here to download Supplementary Material: Supp_Inf.pdf \\ Supplementary Material
Click here to download Supplementary Material: Supp_Inf.pdf}

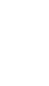

(n)

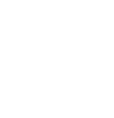

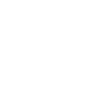

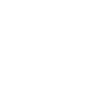

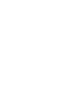

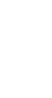

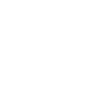
(1)

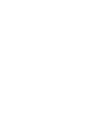

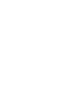

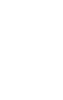

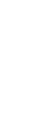

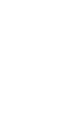

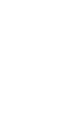

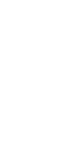

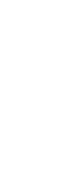
.

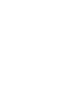



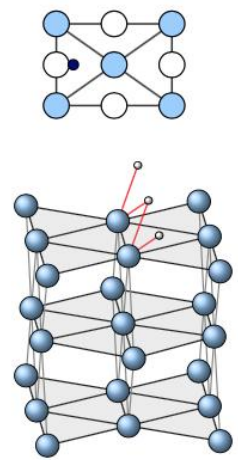

0.5
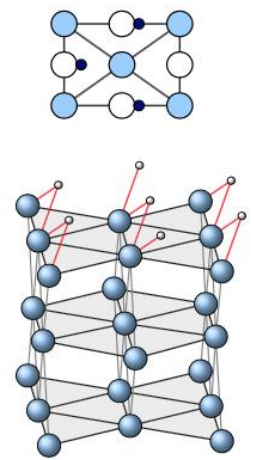

1.0
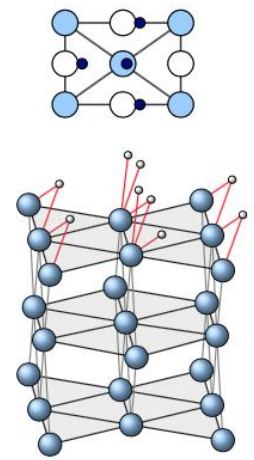

1.5
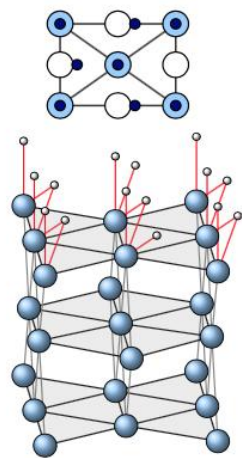

2.0

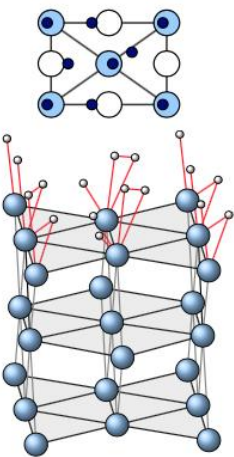

2.5

3 -D visualization of the most stable configurations calculated on the W(110) surface at each coverage ratio investigated. 